ArtefaCToS. Revista de estudios de la ciencia y la tecnología

eISSN: 1989-3612

Vol. 7, No. 2 (2018), 2a Época, 127-144

DOI: http://dx.doi.org/10.14201/art201872127144

\title{
Sobre la dificultad de entender filosóficamente la tecnología
}

\author{
On How Difficult it is to Philosophically Understand \\ Technology
}

\section{Alberto CUPANI}

Universidade Federal de Santa Catarina, Brasil

cupani@cfh.ufsc.br

Recibido: 15/03/2018. Revisado: 19/03/2018. Aceptado: 07/04/2018

\section{Resumen}

La Filosofía de la Tecnología tiene ya más de medio siglo como disciplina académica, pero los autores no concuerdan en una definición común, lo que parece comprometer el propósito de entender filosóficamente la tecnología. En este artículo se indagan las razones de esa dificultad y se argumenta que ella se debe no sólo a la complejidad de la tecnología, sino también a su omnipresencia, su frecuente invisibilidad y su ambivalencia, a las que debe añadirse la diversidad de posición intelectual de los filósofos. Argumentase también que más importante que una definición común es la conciencia de que la tecnología es una realidad en que estamos inmersos, sin que seamos por eso incapaces de investigarla, y que, recíprocamente, lo que de ella podemos decir objetivamente no agota su significado en nuestra vida. Por último, llámase la atención para el desafío que la Inteligencia Artificial representa para la Filosofía de la Tecnología.

Palabras clave: filosofía de la tecnología; omnipresencia de la tecnología; ambivalencia de la tecnología; tecnología e inteligencia humana; tecnología y valores; tecnología y poder; tecnología y racionalidad.

\begin{abstract}
Philosophy of technology has been in existence for more than half a century as an academic discipline, but authors do not agree on a common definition of technology, which appears to compromise the
\end{abstract}


effort to philosophically understand it. This article examines the reasons for this difficulty and argues that it is due not only to the complexity of technology, but also to its omnipresence, frequent invisibility and ambivalence, as well as the diversity of intellectual position among philosophers. The paper also argues that more important than developing a common definition is the awareness that technology is a reality in which we are immersed without this meaning that we are incapable of studying it, and that reciprocally, what we can objectively affirm about it does not encompass its entire meaning in our lives. Finally, the paper highlights the challenge that artificial intelligence represents for the philosophy of technology.

Keywords: philosophy of technology; omnipresence of technology; ombivalence of technology; technology and human intelligence; technology and values; technology and power; technology and rationality.

\section{Introducción}

La Filosofía de la Tecnología ha dejado de ser una disciplina reciente, en vías de consolidación como área del saber académico. Hace ya más de medio siglo desde que Mario Bunge, uno de los pioneros de estos estudios, se quejara de la indiferencia de los filósofos profesionales para con el mundo tecnológico (Bunge, 1980, cap. 13). Hoy tenemos cátedras, publicaciones y congresos que atestiguan una creciente y diversificada reflexión sobre la tecnología, a menudo dialogando con investigaciones sociológicas e históricas a su respecto.

Sin embargo, no parece haber unanimidad sobre el objeto de esta nueva disciplina. La disparidad de las definiciones es sorprendente, aun teniendo en cuenta que la Filosofía es multiforme, sin un "paradigma" común que garantice la unanimidad de su vocabulario. ${ }^{1}$ Leemos, según los autores, que la tecnología consiste en la "fabricación y el uso de artefactos", o que ella es "una forma de conocimiento humano dirigida a crear una realidad según nuestros propósitos"; también, que constituye un "conocimiento que funciona (know how)", o que representa "la humanidad trabajando (at work)". Un famoso pensador la percibió como transformación de la Naturaleza en meros recursos para el hombre. Otro, como el modo de vida propio de la Modernidad. Un tercero, la identificó con "la totalidad de los métodos a que se llega racionalmente y que tienen eficiencia absoluta". ${ }^{2}$ Nuestro ya recordado Bunge la define como "el campo de conocimiento

\footnotetext{
${ }^{1}$ Esa multiformidad de la Filosofía no impide que haya, con relación a lo que sea ciencia, por ejemplo, una relativa unanimidad.

${ }^{2}$ Las definiciones mencionadas corresponden, respectivamente, a Mitcham (1994), Skolimowski (1983), Pitt (2000), Heidegger (1997), Borgmann (1984) e Ellul (1964).
} 
relativo al proyecto de artefactos y a la planificación de su realización, operación, ajuste, manutención y monitoración, a la luz del conocimiento científico" (Bunge, 1985, 231). Eso da la impresión de una falla, pues la definición común parece ser el punto de partida y la síntesis de la adecuada comprensión de un asunto.

Una conjetura plausible para explicar esa pluralidad de caracterizaciones consiste en apelar a la complejidad del tema. Sin duda, la tecnología (sea como fuera que la definamos) se nos presenta bajo muy diferentes rostros, desde los teléfonos celulares de los que creemos no poder prescindir hasta las armas atómicas que esperamos nunca ver en acción. Desde los sofisticados aparatos con que diagnostican nuestras enfermedades hasta los no menos sofisticados que nos proporcionan una "realidad virtual". Desde los sistemas de comunicación que nos informan de casi todo cuanto deseamos saber hasta los vehículos que nos transportan velozmente sobre la tierra, por el agua, por el aire, por el espacio. La tecnología está cada vez más presente en la forma como actuamos, nos movemos y somos, puesto que nos hemos vuelto usuarios semivoluntarios de artefactos y seres dependientes de sistemas que configuran lo que vemos como nuestra vida. Sí, la tecnología es una realidad compleja, pero: ¿es que existe una realidad que sea simple? Recelo que no, y que lo que creemos simple es el producto de nuestra ignorancia o nuestra simplificación. ${ }^{3}$ Sea como fuere, tal vez ocurra con la tecnología lo que con el tiempo en la famosa reflexión agustiniana: la sabemos y a la vez, no la sabemos explicar.

Pero mi propósito en este trabajo no es rendirme a esa conclusión, sino indagar las causas o razones por las que resulta difícil identificar la tecnología en general, aunque sea fácil reconocerla en alguna manifestación particular, como una película, un dron o un corazón artificial. Trataré de aclarar esa dificultad refiriéndome a sus múltiples modos de existencia (primera razón de su complejidad) y a algunos vínculos significativos de la tecnología, para ensayar finalmente una respuesta a la cuestión planteada.

\section{La tecnología y sus modos de existencia}

El filósofo Carl Mitcham, en el libro que constituye tal vez la mejor introducción a la Filosofía de la Tecnología (Mitcham, 1994), hace ver que la tecnología no consiste solamente en objetos y sistemas de ellos, sino también en formas de conocimiento, varios tipos de actividades y en una determinada actitud humana con relación a la realidad, natural o social.

Los objetos tecnológicos son muy diversos: dispositivos, como un GPS, pero también un cierre de cremallera, herramientas, instrumentos (profesionales, deportivos, musicales), estructuras como casas y puentes, máquinas, autómata, así como los sistemas a que pertenecen: eléctricos, hidráulicos, de comunicación,

\footnotetext{
${ }^{3}$ Sobre a complejidad de la tecnología, véase Cupani (2014).
} 
financieros, bélicos, etc. El conocimiento tecnológico (un know-how que no excluye su formulación como know-that) está constituido por habilidades sensorio-motrices, máximas técnicas, reglas de acción que se fundamentan en leyes empíricas y teorías sobre la índole y el funcionamiento óptimo de artefactos y sistemas (Cupani, 2006). Las actividades en que se encarna la tecnología son igualmente diversas: el design o proyecto de lo que se desea, su ejecución y eventual corrección, la producción y testeo del producto, su monitoración, su uso y su reparación, son formas de acción en que nos insertamos en el mundo tecnológico. ${ }^{4}$ Por fin, la tecnología representa una determinada actitud humana frente a la realidad, diferente de otras (como la actitud científica, la religiosa, la artística o la filosófica) que ha sido interpretada diversamente por los filósofos: como voluntad de poder, como busca de eficiencia, como ansia de libertad, como deseo de controlar las circunstancias en que actuamos, como búsqueda de una vida mejor (Mitcham, 1994, 254 ss.).

Ahora bien: aunque es fácil identificar ciertos objetos como tecnológicos (digamos, un aparato de televisión), en otros casos no lo es tanto. Un par de anteojos, por ejemplo, puede no parecer algo tecnológico hasta que reparamos en los conocimientos de óptica que supone su fabricación. Aún menos tecnológico parecen una simple tijera o el vidrio de nuestras ventanas, aunque no existirían sin los procesos industriales (cada vez más automatizados) de los que resultan. Es claro que, de acuerdo con esta última observación, pocos objetos de las sociedades “avanzadas" no son tecnológicos.

Lo mismo ocurre con las actividades tecnológicas. Todas ellas (hasta la producción de autómata, si hemos de creer que Leonardo da Vinci inventó algunos) pertenecen al campo de la artesanía, pero hoy en día asumen una sofisticación cada vez mayor. Los designers trabajan con computadoras; los testeos de prototipos son a menudo virtuales; la producción es fabril; las reparaciones requieren conocimientos teóricos (hasta el electricista debe tenerlos) e implican la aplicación de otros dispositivos tecnológicos. El mero uso de tecnologías exige de nuestra parte, no tanto conocimiento de su funcionamiento (generalmente basta saber "apretar el botón"), sino una opción: por recursos más eficientes, que facilitan nuestras actividades y posibilitan cosas otrora soñadas: volar, evitar enfermedades, comunicarnos a distancia...

En cuanto a la tecnología como actitud humana, es indudablemente tentador entenderla como algo inherente a toda producción tecnológica. Dos de esas interpretaciones son particularmente seductoras: la que ve en la tecnología cierta voluntad de poder (Mumford, 1963), y la que la explica por el deseo de vivir mejor (Ortega y Gasset, 1965). Sin embargo, ellas son difíciles de aplicar a todas

\footnotetext{
${ }^{4}$ La inmensa mayoría de nosotros, tan sólo como usuarios y eventualmente víctimas de las fallas de funcionamiento de los artefactos, o de nuestra incompetencia.
} 
las formas y los casos de tecnologías. ¿Es para vivir mejor que se fabrican armas cada vez más mortíferas? ¿Es una máquina de lavar ropas un instrumento de poder?

\section{Tecnología y técnica}

Aunque objetos y sistemas sean las manifestaciones tecnológicas que más fácilmente evocamos, la tecnología existe más primariamente en formas de actividad estandarizadas y por ende, transmisibles, que nos permiten producir un resultado deseado de manera económica en términos de esfuerzo, tiempo y recursos. O sea, ella existe en forma de técnicas. Los artefactos (ya sea un cuchillo o una perforadora eléctrica) y los sistemas (como el arco y su flecha, o una usina) vienen a ser, como alguien dijo, la encarnación o concreción de un método, de una forma confiable de producir ciertos efectos (Ferré, 1995, 37).

La vida propiamente humana es impensable sin técnicas. Aunque no reflexionemos sobre ello, caminar y hablar son técnicas sin las que no seríamos lo que somos. Igualmente el hablar español, en vez de mandarín. En la medida en que no somos organismos puramente instintivos, ${ }^{5}$ nos servimos constantemente de técnicas que asimilamos desde la infancia, irreflexivamente, y de otras que fuimos y vamos adoptando según nuestros intereses y necesidades. Actuamos técnicamente al cocinar, al nadar, al tocar un instrumento y, por supuesto, al calcular. Lo hacemos al sembrar, al dar una inyección y al andar a caballo. Algunas técnicas son (o parecen, una vez adquiridas) muy fáciles, como coser un botón en nuestra ropa. Otras son más difíciles, exigiendo de nuestra parte más entrenamiento y volviéndose eventualmente dominio de peritos (como bailar a la perfección, o regular el funcionamiento de un motor). Es en estos casos donde parece más adecuado hablar de tecnología y no de (mera) técnica). Tanto una vela cuanto una lámpara eléctrica encarnan técnicas de iluminación, del mismo modo que tanto un carro de bueyes cuanto una camioneta encarnan técnicas de transporte, y un puñal y un rifle de mira telescópica encarnan técnicas de ataque y defensa. En cambio, pilotar un avión, filmar una película o usar una impresora tridimensional no parecen tener antecedentes semejantes. Es decir, las que intuitivamente consideramos como tecnologías (posiblemente, atentos a sus aspectos más alejados de nuestras habilidades corporales), no dejan de ser técnicas. Esto es: procedimientos (y artefactos que los concretizan) que nos permiten alcanzar sistemáticamente objetivos deseados. Con otras palabras: tecnologías serían cierto tipo de técnicas, tal vez las particularmente sofisticadas, o insólitas, o las que más amplían o superan las capacidades humanas. Aún con otras palabras: entre técnica y tecnología no habría sino una diferencia de grado. ${ }^{6}$

\footnotetext{
${ }^{5}$ Por eso, hay también técnicas desarrolladas por animales que parecen superar ocasionalmente la conducta instintiva.

${ }^{6}$ Es por eso que algunos autores hablan de "técnicas", genéricamente, y otros de "tecnologías", 
Pero, ¿es lo mismo iluminarse con una vela y con una lámpara eléctrica? ¿Es lo mismo transportar una carga en un carro y en una camioneta? ¿Es lo mismo atacar o defenderse con un puñal o con un rifle? Algo nos dice que no, o mejor: que es y no es lo mismo, en cada caso. Pues, si por un lado alcanzamos el mismo objetivo con una técnica tradicional y con una tecnología, por otro, el recurso a esta última parece modificar más la acción humana, sus frutos, el mundo humano así resultante. No es la misma experiencia la de un local iluminado eléctricamente que a vela; tampoco lo es transportar una carga en carro o en camioneta; ni herir (y eventualmente, matar) a otro ser vivo cuerpo a cuerpo en vez de a distancia.

\section{Tecnología e inteligencia}

Fáciles o difíciles, las técnicas implican un saber hacer, esto es: actuar técnicamente es lo contrario de actuar ciega o mecánicamente. ${ }^{7}$ Aunque procedamos "sin pensar" (por ejemplo, a abrir una puerta girando en una dirección la llave en la cerradura, o al pedalear en una bicicleta), siempre podemos dar razón de que así debemos proceder, y de ese modo podemos enseñar a otros una técnica (por más que al hacerlo nos descubramos malos maestros, como pasa al racionalizar una conducta "espontánea").

¿Qué tiene esto que ver con la tecnología? Pues que ella se deja percibir, sea en la substitución de unas técnicas por otras, más sofisticadas (por ejemplo, el abanico por el ventilador eléctrico) o por la aparición de técnicas que permiten lo que antes no era posible (como el avión o el submarino). Si toda técnica amplía el horizonte de posibilidades de la vida humana, algunas parecen tornar evidente que esa ampliación es indefinida y siempre sorprendente. Escribíamos con plumas, lápices y (¡oh, maravilla!) con la máquina de escribir. Ahora lo hacemos tecleando en nuestra computadora ( $y$ en breve, tal vez apenas pensando en lo que queremos decir). Nos comunicábamos por carta: hoy lo hacemos mediante el WhatsApp. Andábamos sobre la tierra y navegábamos: hace tiempo que podemos volar, y es posible que vayamos a otros planetas.

El que denominemos "inteligentes" ciertas tecnologías, enfatiza una trivialidad: técnicas y tecnologías surgieron de la inteligencia humana. O mejor: de dificultades vitales que la desafiaron. En este tema, me gusta la definición de Frederick Ferré, inspirado en Whitehead): inteligencia es la capacidad de disciplinar la mente para lidiar con problemas, prácticos o teóricos (Ferré, 1995, cap. 4). La técnica es producto de la inteligencia puesta al servicio de la necesidad y el deseo humanos de sobrevivir y mejorar la existencia. La tecnología surge cuando la inteligencia

del mismo modo.

${ }^{7}$ Ya los griegos denominaban tekhne al saber hacer (producir). 
teórica, en cuanto capacidad de tratar abstractamente de problemas y aumentar la comprensión crítica del mundo, es puesta sistemáticamente al servicio de la inteligencia práctica.

No puede sorprender que, traduciendo inteligencia teórica por ciencia e inteligencia práctica por técnica, haya sido frecuente entender la tecnología meramente como ciencia aplicada. Sin embargo, hoy se reconoce que la tecnología "aunque pueda aplicar ciencia, no es lo mismo que, o enteramente, ciencia aplicada”, en la feliz fórmula de Walter Wincenti, ingeniero y teórico de la ingeniería (Vincenti, 1990, passim). Eso, no sólo porque diversas tecnologías surgieron antes de la ciencia moderna, se anticiparon a conocimientos científicos o hasta los originaron (como en el conocido caso de la máquina de vapor con relación a la termodinámica), sino porque la tecnología implica modos específicos de saber, diferentes del saber científico, como fue mencionado.

Pero la relación de la tecnología con la inteligencia es aún más estrecha. Según Andy Clark (2003), la inteligencia humana nunca estuvo contenida o limitada por el cerebro humano, o por nuestra corporalidad. A diferencia de los animales no humanos, nuestra mente está abierta a combinaciones múltiples con herramientas, ayudas y apoyos. Somos así "naturalmente ciborgs" (organismos cibernéticos). Fue sobre todo a partir de la adquisición del lenguaje, de la escritura y de la imprenta, argumenta Clark, que el pensamiento humano se exteriorizó, y lo que llamamos mundo es, cada vez más, un sistema de instrumentos y sostenes tecnológicos para nuestro pensamiento, nuestra acción, nuestras reacciones, nuestros sentimientos. ¿Qué decir de esa simbiosis desde que existen los mecanismos automáticos, la red global, la inteligencia artificial? El mundo tecnológico es un sistema inteligente con el que interactuamos. A su vez, Davis Baird (2004) señala que los artefactos (tanto una regla como un libro o un microscopio) no son meros portadores o vehículos de conocimiento, sino que lo encarnan. Todo ello implica que la tecnología no es una entidad externa a los seres humanos, sino algo que forma parte de nuestro "ser-en-el-mundo". Razón tiene, pues, un fenomenólogo como Don Ihde al reivindicar su enfoque teórico para evitar concebir la tecnología como un "objeto" que estuviera "ante" nosotros (Ihde, 1990, esp. Cap. 2).

\section{Tecnología y poder}

Sin perjuicio de lo anteriormente dicho, es indudable que la tecnología, si no siempre, tiene generalmente que ver con el ejercicio de alguna forma de poder (o el acceso, o la resistencia al mismo). Me convence la tesis heideggeriana de que la técnica moderna representa la voluntad humana de que la Naturaleza se reduzca a recursos a disposición del hombre (Heidegger, 1997). Puede complementársela con la tesis de Albert Borgmann según la cual la tecnología moderna significa el triunfo del "paradigma del dispositivo", o sea de la mentalidad para la cual todo 
es algo-para propósitos humanos (Borgmann, 1984, esp. cap. 9). ${ }^{8}$ Esa visión se emparenta con las críticas (las más famosas, las de la Escuela de Frankfurt) al uso de la tecnología, en la sociedad industrial, como medio de forzar el consenso social y evitar toda resistencia. Eso se haría sugiriendo que la vida más cómoda y fácil gracias a la tecnología es también la más democrática, como en el conocido (Marcuse, 1982). Pero esa manera de entender el poder (esto es, como dominio) olvida su otra dimensión: el poder como capacitación. Las técnicas primero, y las tecnologías después hicieron que individuos y grupos pudieran más y mejor. Alimentarse más convenientemente, evitar enfermedades y curarse de ellas, desplazarse más fácilmente, comunicarse a través del espacio y del tiempo. También, que fueran capaces de resistir a la opresión y la manipulación. ${ }^{9}$ En otras palabras: la tecnología ha sido, y continúa siendo, factor tanto de sujeción (de la Naturaleza al hombre, de unos hombres a otros) como de liberación o mejoramiento de condiciones de vida. Lo que ocurre en los casos particulares no puede generalizarse sin evocar contraejemplos.

Por otra parte, en nuestro ${ }^{10}$ satisfecho disponer de smartphones, vehículos cada vez más automatizados, información instantánea e infinita a través de la Internet, viajes aéreos y medicamentos que nos mantienen sanos, olvidamos que esa capacitación tiene un precio o un reverso. Dependemos cada vez más de ellos, y nos entregamos a todo tipo de manipulaciones (disimuladas o no) y de riesgos. Me refiero a informaciones o noticias falsas, a la desorientación por el exceso de datos, a decisiones que son tomadas por los sistemas que gobiernan los artefactos, a consecuencias no sabidas de los remedios, a agresiones al medio ambiente de que somos involuntariamente cómplices, etc.

La tecnología tiene, sí, relaciones con el poder, pero son camaleónicas. En todo caso, esas relaciones hacen de ella algo obviamente político. Langdon Winner fue uno de los primeros pensadores de la tecnología en explorar el carácter potencial o efectivamente político de sus diversos tipos (Winner, 1986). Desde las que "exigen" una organización política centralizada (como las usinas atómicas), a las que favorecen formas de vida democrática, como la energía solar, pasando por las que tienen consecuencias políticas no intencionales (como las instalaciones que ignoran la existencia de zurdos o lisiados, y la robotización industrial que elimina empleos). Prima facie parece exagerada su fórmula de que "los artefactos tienen políticas", pero poca reflexión es necesaria para concluir que la existencia

\footnotetext{
${ }^{8}$ Cuando se repara (aunque prefiramos no hacerlo) en la manera despiadada en que la industrialización de la producción agropecuaria trata plantas y animales (desde la producción de cereales transgénicos al confinamiento brutal de los animales para extraerles provecho), poca duda queda de que la aparición del homo sapiens representó, en palabras de Yuval Harari, una catástrofe terrible para los seres vivos no humanos (Harari, 2016, cap. 4).

${ }_{9}^{9}$ Piénsese en ese papel de la imprenta desde sus comienzos y en el de las redes sociales actualmente.

10 "Nuestro" con las debidas licencias. No me refiero, es claro, a los marginalizados de "nuestras" sociedades.
} 
de determinadas tecnologías y no otras manifiesta el predominio de cierta política (económica, sanitaria, bélica, etc.). Y que esa misma existencia impide generalmente la imaginación de otras tecnologías, quizás mejores.

\section{Tecnología y valores}

Toda actividad humana se orienta, implícita o explícitamente, por y para valores. ${ }^{11}$ Responde a lo que estimamos importante, necesario, agradable, bello, etc., y refuerza la constelación de lo que, individual y comunitariamente, se admite como valedero y hasta imperativo (ya sean la honestidad, la solidaridad y la justicia, o el éxito, el lucro, el placer).

¿A qué valores responde la tecnología? Como resultante de proyectos humanos, puede decirse que a cualquier valor (o desvalor): tanto a la salud como a la destrucción, tanto al avance del conocimiento (en particular, a la ciencia) como a la vigilancia ideológica, y tanto a la creación artística como a la censura. ¿ Significa ese reconocimiento que adherimos a la noción común de que la tecnología es neutra, y que todo depende de las intenciones de creadores, productores y usuarios? A pesar de ser seductora, y de tener sus defensores en materias delicadas ("no son las armas, sino las personas quienes matan"), no debemos precipitarnos a aceptarla. La tecnología, en abstracto, puede parecer neutra, pero ella existe, en concreto, en contextos que la hacen no-neutra (en líneas generales, entre benéfica y perjudicial).

En todas sus manifestaciones, la tecnología parece responder al deseo de proceder eficientemente. Eficiencia significa alcanzar un efecto deseado (eficacia) de manera económica: evitando desperdicios de esfuerzo, materiales, tiempo y dinero. ${ }^{12}$ Pero "eficiencia" es un término comparativo y contextual. Ella varía conforme las actividades y profesiones, siendo apreciada por relación con versiones anteriores de un artefacto (teléfono, perforadora, motocicleta) o a la inexistencia de ese tipo de instrumento, como al ser inventada la fotografía. ${ }^{13}$ En todo caso, la eficiencia está ligada a la economía y a la facilidad como valores inherentes a la tecnología. También al control de las circunstancias y recursos cuando se quiere alcanzar una meta y a la previsibilidad (ambos valores, también intrínsecos a la técnica). Casi siempre, un nuevo recurso tecnológico vuelve más rápida una operación. Y en alguna medida, toda nueva tecnología es precisamente eso: algo

\footnotetext{
${ }^{11}$ Discúlpeseme esta presentación simplificada, que apela a nociones intuitivas, de un tema tan complejo.

${ }^{12}$ La noción de eficiencia y su cultivo como valor, tienen una interesante historia, especialmente en la forma como, originándose en la ingeniería, la noción emigró para las actividades sociales (Alexander, 2009).

${ }^{13}$ Para la reproducción supuestamente fiel de imágenes.
} 
nuevo y apreciado como tal. Por fin, la substitución de una tecnología por otra es considerada un progreso con relación a la manera anterior de proceder, algún tipo de liberación con relación a tareas difíciles de cumplir. ${ }^{14}$

Eficiencia, control, economía, facilidad, rapidez, novedad, libertad, progreso: creo que esos valores están en el núcleo de toda tecnología. Ciertamente que el peso relativo de cada uno de ellos (y hasta el significado de "eficiencia") depende de los contextos concretos de acción humana. La eficiencia (o mayor eficiencia) de una vacuna no es definida ni apreciada del mismo modo que la eficiencia de una transmisión televisiva o la de una campaña militar. ${ }^{15}$

Lo anterior significa que, al optar por usar determinada tecnología o producirla, estamos decidiéndonos por cursos de acción, y hasta por cierta visión del mundo en términos de metas que han de ser alcanzadas de forma relativamente segura, una vez que estemos convencidos de la importancia (o necesidad) de esas metas, o que no las discutamos, o (peor aún) que no las podamos discutir. ${ }^{16}$

La sociedad occidental de los siglos XX y XXI es la de la universalización de la eficiencia como forma preferible de conducir la vida. Toda novedad nos es impuesta o vendida bajo alegación de su mayor eficiencia (que acarrea, supuestamente, los otros valores: economía, facilidad, rapidez, liberación, etc.). Pero es claro que de la disponibilidad de una nueva tecnología no debemos deducir que sea más eficiente que la anterior, o que sea imprescindible. Sería una inferencia tan ingenua como falaz. Sin negar que sea ése en muchas ocasiones el caso, en otras la alegada mayor eficiencia se reduce a un truco propagandístico, comercial o político. ${ }^{17}$

Los demás valores inherentes a la tecnología suscitan también dudas filosóficas. El control de las circunstancias nunca es total, de lo que derivan consecuencias no siempre deseadas. La economía de algunos recursos, como el esfuerzo, puede pagarse con dańos, como los producidos por el sedentarismo de una vida acostumbrada al automóvil, el ascensor, la escalera mecánica. La facilidad en la operación trivializa lo que costó implementar (parécenos que la Internet nació con nosotros). La rapidez habitual nos deja impacientes, como se puede observar en los usuarios de juegos electrónicos. Toda novedad parece agotarse rápidamente, pidiendo otra. Nos libertamos tecnológicamente al precio

\footnotetext{
${ }^{14}$ En el sentido en que una retroexcavadora, por ejemplo, evita la penosa tarea de cavar con palas.

${ }^{15}$ Feenberg $(2002,78-79)$ sugiere que, en una economía diferente de la capitalista, la línea de producción, forma aparentemente excelente de organizar la fabricación de artefactos, podría no constituir la tecnología más eficiente.

${ }^{16}$ Es lo que ocurre, en la sociedad globalizada, con metas como estar siempre comunicado e informado, "acompañar el progreso" o vivir más tiempo.

${ }^{17}$ Jennifer Alexander señala que "la historia de la eficiencia revela una profunda y larga asociación con el poder y la autoridad”, sobre todo a partir del industrialismo (Alexander 2009:1007, traducción mía).
} 
de depender de la manutención de los artefactos, de su recarga, de la ayuda de peritos, sin hablar del endeudamiento para adquirir y mantener los dispositivos. Creemos vivir por y para el progreso, pero las condiciones de esa vida (como el ansia de "estar al día" tecnológicamente) y ciertos efectos de ella (como la polución ambiental y el abuso de la Naturaleza) nos hacen añorar una vida más simple.

\section{Tecnología y racionalidad}

Además, ¿por qué elegir la eficiencia como la mejor manera de actuar en cualquier circunstancia? Se dirá que porque es la manera más racional, si por tal cosa entendemos la elección de los medios (disponibles o imaginados) más apropiados para alcanzar determinado fin. Pero, como ha sido observado tantas veces, el fin en cuestión es dado al hacer tecnológico, que representa por eso una racionalidad (meramente) instrumental o pragmática (Agazzi, 1996, 211). Curar un ser humano, enseñarle algo o divertirlo son fines eventualmente propuestos a la tecnología tanto como condicionar ese mismo ser (por ejemplo, mediante la propaganda) o torturarlo. Con frecuencia, esa forma de acción racional supone la ausencia de sensibilidad o emotividad (como al explotar industrialmente los animales) o su complemento por otra forma de acción eficiente (por ejemplo, al anestesiar un paciente para operarlo).

La tecnología encarna o representa una forma de pensar y actuar que parece racional por antonomasia. Pero se puede ser "racional" tanto en la salud mental benévola como en la locura sádica. Además, ¿por qué creer que todo problema humano tiene una solución técnica? Es dudoso que problemas morales, políticos y existenciales la tengan.

La tecnología responde al llamado "imperativo tecnológico" ("lo que puede ser producido, ha de serlo"). Ese imperativo constituye lo que podríamos llamar su inercia, a ser facilitada o impedida por factores ambientales (materiales o sociales). ${ }^{18}$ Una vez existente, toda tecnología tiene una manera correcta y normal de funcionar, ya se trate de un destornillador, un motor o un hospital. Aquel imperativo y este funcionamiento son las únicas exigencias (deber-ser técnico) propias de la tecnología, que pueden ciertamente ser contrariadas: un artefacto o sistema puede no llegar a ser producido, puede ser usado indebidamente (el caso típico del destornillador utilizado como abrelatas), puede funcionar mal o descomponerse.

Ahora bien: en una sociedad cada vez más tecnológica, las personas pasan a "funcionar" y ser juzgadas de manera análoga a los artefactos. También ellas (nosotros) "deben" actuar (vivir) de cierta manera, pudiendo asimismo ser mal

\footnotetext{
${ }^{18}$ Me refiero a la existencia o falta de determinada materia prima, por ejemplo, o a intereses favorables o contrarios a su producción.
} 
"utilizadas", no "funcionar" como de ellas "se espera" por ser elementos de tal o cual sistema (económico, político, jurídico, pedagógico, etc.), o dejar de operar (por accidente, desempleo, enfermedad, muerte). ${ }^{19}$

Pero la racionalidad humana refiérese también, y sobre todo, a nuestra capacidad de examinar y elegir fines o metas de nuestras acciones y de nuestra vida. ¿Nos es necesaria determinada tecnología? ¿Vale la pena substituir este aparato por su nueva versión? ¿Debe ser producido todo lo que puede serlo? ¿En qué ayudarán tales o cuales recursos tecnológicos para que seamos fieles a los valores intelectuales, éticos o religiosos que profesamos? ¿Es una sociedad tecnológica más o menos democrática? ¿Hay una tecnología del arte o de la felicidad? Esas y otras preguntas colocan la tecnología en las perspectivas del sentido de nuestra existencia, de nuestra responsabilidad moral y de nuestra vida ciudadana. Ellas tienen que ver con la racionalidad práctica (y no meramente pragmática) de nuestra acción (Agazzi, 1996, esp. cap. IX), amenazada por la vida y la mentalidad tecnológicas porque ellas pueden suprimir la concepción de alternativas (Postman, 1993).

\section{Ensayando una respuesta}

Los vínculos de la tecnología con los factores antes elegidos no agotan, ciertamente, su posible análisis. Podríamos explorar también (sólo para mostrar algunas posibilidades) las cuestiones éticas que ella suscita, como la licitud de ciertos procedimientos médicos o farmacéuticos, ${ }^{20}$ de transformaciones del medio ambiente y del uso de las TICs. O podríamos examinar los diversos modos en que la tecnología condiciona nuestra experiencia, tan bien mostrados por Ihde (1990, esp. cap. 5). Y podríamos, es claro, recordar que nos las habemos con tecnologías que existen more capitalista, y que no podemos saber, mientras dure el capitalismo, qué formas y consecuencias ellas tendrían bajo otro sistema de producción (y de vida), a no ser sospechando que podrían ser bien diferentes.

Pero el análisis de esas y otras relaciones de la tecnología no son, a mi ver, imprescindibles para advertir lo que ya puede constatarse por la exposición precedente: la tecnología no es sólo una realidad compleja (lo habíamos admitido de antemano), sino también algo ubicuo y también ambiguo (o si se prefiere, ambivalente).

La tecnología está en todas partes, en la sociedad occidental actual (y poco a poco, por efecto de la globalización, en las otras culturas). Tanto, que

\footnotetext{
${ }^{19}$ Hasta nos vemos a nosotros mismos como aparatos, al "acceder" a recuerdos "almacenados" en nuestra mente, y "programarnos" para cumplir nuestras tareas.

${ }^{20}$ Las transfusiones, los implantes y la píldora anticoncepcional (recursos dirigidos a salvar vidas, mejorarlas o evitar otros problemas) pueden plantear dilemas morales al paciente, según sus creencias.
} 
generalmente pasa desapercibida. Se volvió algo "natural", ${ }^{21}$ y como que transparente, en gran medida por fuerza del hábito: a nadie sorprenden ya las heladeras, las inyecciones o los helicópteros. La ignorancia o desatención tienen igualmente su parte en esta falta de percepción: nuestra ropa y nuestra vajilla son testigos de la tecnología que las produjo, no menos que nuestro reloj. Pero contribuye a que no siempre notemos la tecnología el que a veces sus efectos sean imperceptibles a la sensibilidad común (como los diversos tipos de radiaciones que emiten los artefactos, posibilitando cocinar, divertirse, curar, matar), que estén dentro de nuestro cuerpo (como los lentes de contacto, el marcapasos o el efecto de los remedios) o que funcionen como trasfondos de nuestra existencia (el aire acondicionado, la iluminación, la música ambiental). ${ }^{22}$ En resumen: nuestro mundo es literalmente tecnológico. La tecnología es nuestro medio, y sólo a través de ella podemos contactar lo no-tecnológico: la Naturaleza, y esas extrańas realidades llamadas amor, belleza, libertad, verdad, justicia, nuestro yo, la divinidad. ${ }^{23}$ La tecnología es nuestra atmósfera, y como ella, difícil de reconocer, a no ser que falte o se enrarezca. Esa omnipresencia dificulta, creo, una definición satisfactoria para todos los estudiosos. Tanto cuantitativa como cualitativamente, unos privilegian ciertas manifestaciones de la tecnología y otros, otras. Y todos ellos acaban por tener dificultades al delimitar (definir) lo que entienden por tecnología con relación al resto "no tecnológico" de su visión del mundo: casi todo lector encuentra excepciones y casos dudosos.

Por su parte, la ambigüedad o ambivalencia de la tecnología ha podido ser observada, espero, en los numerosos ejemplos que fui citando. La tecnología es y no es mera prolongación de la técnica. Parece formar parte del mundo exterior a nuestros cuerpos y mentes, pero acaba siendo interna a ellos. Nos capacita para obrar mejor y para tener nuevas experiencias, pero también nos condiciona, subyuga, amenaza. Gracias a ella, somos más eficientes, pero corremos el riesgo de que nuestra vida se reduzca a ese modo de ser, identificado con una racionalidad que parece ignorar, excluir o asimilar preocupaciones no tecnológicas. No por último, la tecnología parece a menudo reducirse a aquellos aparatos y efectos más ajenos a la comprensión del lego, como los cohetes espaciales y los trasplantes de órganos, pero a veces nos damos cuenta de que pocas cosas no son tecnológicas en nuestra vida cotidiana.

La tecnología es, pues, en sus manifestaciones, innegablemente compleja, omnipresente y ambivalente. ¿Cómo sorprenderse de que sea difícil hallarle una definición consensual? Nótese también que podemos, con igual convicción,

\footnotetext{
${ }^{21}$ Creando, como es sabido, dificultades crecientes para distinguir lo natural de lo artificial.

${ }^{22}$ La nanotecnología se encargará, parece, de que esa presencia ausente sea aún más poderosa.

${ }^{23}$ Espero que el lector o la lectora crea al menos en algunas de ellas.
} 
hablar de la tecnología o de las tecnologías, sin buscar en ellas una esencia común, a no ser que filosofemos. ${ }^{24}$ Nos desafía, pues, una realidad de la que ni siquiera podemos garantizar que sea única cuando sobre ella meditamos.

A los anteriores motivos podríamos sumar la notoria variedad, ya mencionada, de estilos de reflexión filosófica existentes: analítico, fenomenológico, hermenéutico, pragmático, neomarxista, etc., sin olvidar sus combinaciones, una variedad que vuelve todavía más difícil la coincidencia de los pensadores en una definición común de tecnología. Ampliando la visión de este asunto, Mitcham (1994, cap. 3) distingue dos tipos "en tensión" de filosofía da tecnología: la de los "ingenieros" y la de los "humanistas". ${ }^{25}$ Por la primera, entiende la forma de analizar la tecnología propia de aquellos pensadores de espíritu más afín a ella y a la ciencia, y que a veces tienen formación científica o tecnológica. Su enfoque se orienta a caracterizar, clasificar y explicar rigurosamente las diversas formas de tecnología y tienden a percibir los ámbitos y problemas que la transcienden (ética, política, arte, literatura, religión...) en términos de ella misma. La segunda estaría representada por los autores pertenecientes a las humanidades (filósofos tradicionales, sociólogos, historiadores, literatos, pedagogos), que se interesan por la relación de la tecnología con lo "trans-técnico" y desean sobre todo comprender la tecnología de un modo hermenéutico, en sentido amplio. ${ }^{26}$ Los "ingenieros" tienden a percibir la tecnología en sus aspectos más positivos, como manifestación e instrumento del progreso humano, mientras que los "humanistas" prestan más atención a las incertezas y riesgos de la civilización tecnológica. Esa diferencia de perspectiva, no siempre transparente en las definiciones propuestas por los diversos pensadores, conspira también contra una caracterización de la tecnología que sea convincente para todos. ${ }^{27}$

Por todos esos motivos, poca esperanza queda de que pueda llegarse algún día a una definición unánime de la tecnología. Pero, ¿será eso tan importante? La falta de consenso sobre una definición no impide a los filósofos indagar sobre

\footnotetext{
${ }^{24}$ Algo semejante ocurre con el arte o la cultura, pero no con "caballo" o "tenedor". Tal vez debamos contentarnos con una caracterización à la Wittgenstein, admitiendo que "tecnología" alude solamente a un "aire de familia", y que nos referimos a las tecnologías en un determinado "juego de lenguaje” (Wittgenstein, 1979)

${ }^{25}$ Aquí, "filósofo" tiene, ciertamente, un sentido más amplio, denotando aquellos estudiosos con preocupaciones típicamente filosóficas, y no filósofos profesionales.

${ }^{26}$ En sus extremos, pienso yo, tendríamos de un lado un Bunge, físico y filósofo, que llega a proponer una ética científicamente formulada (Bunge, 1989), y de otro, un Heidegger (1997), metafísico aun cuando se quería post-metafísico, pronunciándose sobre a "esencia no técnica" de la técnica (tecnología).

${ }^{27}$ Puede coincidirse en la letra, pero no en el espíritu de una definición, que sólo se entiende bien al conocer la obra de la que tal definición es (o debería ser) un epítome. Es entonces cuando surgen los argumentos que vuelven difícil la coincidencia de los autores.
} 
asuntos tales como el conocimiento humano o el deber moral. Más importante me parece meditar sobre lo que pueda significar "entender" filosóficamente la tecnología. ¿Qué tipo de reflexión sobre la tecnología es posible y necesaria?

Sugiero que los motivos anteriormente presentados para explicar la dificultad de alcanzar una definición universalmente admitida de la tecnología nos fuerzan a reconocer que ella no se "coloca" ante nosotros como un objeto que pudiésemos reconocer y diferenciar de los otros, tantos, que constituyen nuestra experiencia de lo que, bien o mal, llamamos realidad. Estamos, como recordé antes, inmersos en ella, y su problematización filosófica parece remitir a lo que el hoy olvidado Gabriel Marcel decía de los problemas filosóficos: son una suerte de misterio que forma parte de nosotros. En este sentido, sería bueno que los pensadores "ingenieriles" no estén tan seguros de que la tecnología consiste en lo que podemos decir de ella objetivamente, y que explicar sus manifestaciones no agota lo que sobre ella podemos pensar.

Lo que denominamos tecnología tiene que ver con los aspectos en que nuestra condición como seres humanos parece superar, constantemente y por su propia dinámica, los que teníamos por sus límites (y limitaciones), para mejor o para peor, con el agravante de que la tecnología parece complicar y confundir nuestras nociones de lo "mejor" y lo "peor". Este es un reto para los pensadores "humanistas", a menudo asustados por las que les parecen amenazas de la tecnología a lo "propiamente humano" y dados a temer la tecnología como una fuerza autónoma.

Creo también que un adecuado entendimiento de la tecnología exige que los "ingenieros" presten más atención, por ejemplo, a los nuevos problemas éticos, ecológicos y políticos por ella suscitados, que no se reducen a problemas tradicionales en mayor tamaño, ni pueden ser resueltos por análisis de gabinete, sin discusión pública. ${ }^{28} \mathrm{~A}$ su vez, los "humanistas" deben tratar de indagar si lo que parece una pérdida o distorsión de la condición humana no encierra una nueva forma de realización de la misma, para lo que frecuentemente deberán familiarizarse con cuestiones técnicas, asumiendo circunstancialmente la actitud científico-tecnológica del investigador que pone un objeto bajo su lente. ${ }^{29}$

Además de cambiar de actitud, "ingenieros" o "humanistas" deberían aprender a dialogar entre sí. La filosofía, al final de cuentas, es diálogo o no es nada. La visión de los "humanistas" como "poco informados" o hasta "irracionales", o la de los "ingenieros" como como "positivistas" o "ingenuos" no ayuda al necesario intercambio de ideas y puntos de vista sobre una cuestión (es una manera de

\footnotetext{
${ }^{28}$ Me refiero a cuestiones como los cambios de sexo, la responsabilidad para con las generaciones futuras y el control de la vida personal a través de los medios de comunicación.

${ }^{29}$ La distancia epistemológica así practicada ayudaría a disminuir el peso de la tradición cultural que vuelve a los "humanistas" desconfiados ante las novedades tecnológicas.
} 
decir) tan difícil. ${ }^{30}$ Aquí como en cualquier otro caso de indagación filosófica, hay que apelar para la colaboración, confiando más en la argumentación crítica que en la convicción.

Por otra parte, la filosofía de la tecnología no puede reducirse, a una actividad de y para académicos, una certeza compartida por todo autor de un libro o artículo destinado al público en general. Espérase de la filosofía que sea social y culturalmente provechosa. Para eso, es menester que los filósofos participen de actividades extra académicas (foros, asesorías, movimientos políticos) en que puedan ayudar a las instituciones y al público a lidiar con los desarrollos tecnológicos y sus problemas. Esa participación es también una salvaguardia para que el filósofo entienda la tecnología más allá de sus teorías. ${ }^{31}$ No por último, sería una reafirmación del carácter político (en sentido etimológico y no etimológico) que la filosofía occidental tuvo en sus comienzos y mantuvo ocasionalmente (sobre todo, en sus figuras cuestionadoras de ideas e instituciones) en contra de su neutralización como rutina académica.

Sin embargo, la confianza que así deposito en una filosofía de la tecnología que no se encierre en partidarismos ni en su proverbial torre de marfil está hoy amenazada por el avance de la Inteligencia Artificial. De acuerdo con analistas como Yuval Harari (2016b), esa creación tecnológica está cada vez más presente en nuestro modo de vida, volviéndolo progresivamente menos nuestro. Dispositivos, máquinas y sistemas paulatinamente más “inteligentes", que están aprendiendo a aprender, parecen destinados a substituir nuestra capacidad de pensar, decidir, trabajar. ${ }^{32}$

¿Es que será la Filosofía de la Tecnología, en un futuro tal vez no lejano, suprimida o reemplazada por la Inteligencia Artificial (y sus controladores)? Ese, creo, es el principal desafío de la tecnología a la filosofía en este momento, su "descíframe o te devoro". Eso es lo que sobre todo debemos entender y enfrentar.

\section{Referencias bibliográficas}

Agazzi, E. (1996). El bien, el mal y la ciencia. Las dimensiones éticas de la empresa cientifico-tecnológica (trad. de Il bene, il male e la scienza. Le dimensioni étiche dell'impresa scientifico-tecnologica, 1992). Madrid: Tecnos.

\footnotetext{
${ }^{30} \mathrm{Su}$ oposición es una manifestación del desacuerdo entre las "dos culturas", la científica y la literaria, hecho famoso por C.P. Snow en 1959 (Snow, 1995).

${ }^{31}$ En ese sentido, quisiera destacar como ejemplo la actividad del filósofo Hugh Lacey en auxilio de la discusión pública del cultivo de transgénicos en Brasil, para la que publicó un importante libro, proponiendo una plataforma teórica común a defensores y críticos de aquellos productos (Lacey, 2006)

32 Algoritmos se encargan de diagnósticos, defensas judiciales, administración de empresas, inducción de consumo. Programas de computadora derrotan jugadores de diversos tipos de juegos. Otros componen música o manejan automóviles (Harari, 2016b, esp. Cap. 9).
} 
Alexander, J.K. (2009). The Concept of Efficiency: An Historical Analysis, in Meijers, A. (ed.) Philosophy of Technology and Engineering Sciences. Amsterdam: Elsevier: 1007-1029.

Baird, D. (2004). Thing Knowledge. A Philosophy of Scientific Instruments. Berkeley: University of California Press.

Borgmann, A. (1984). Technology and the character of contemporary life: a philosophical inquiry. Chicago: The University of Chicago Press.

Bunge, M. (1980). Epistemologia. São Paulo: T.A. Queiroz/Edusp. (trad. de Epistemología. Barcelona, 1980).

Bunge, M. (1985). Treatise on Basic Philosophy, vol. 7. Dordrecht: D. Reidel.

Bunge, M. (1989). Treatise on Basic Philosophy. Ethics: The Good and the Right, vol. 8. Dordrecht: D. Reidel.

Clark, A. (2003). Natural-Born Cyborgs. Minds, Technologies and the Future of Human Intelligence. New York: Oxford Univ. Press.

Cupani, A. (2006). "La peculiaridad del conocimiento tecnológico", en Scientiae Studia (Brasil) (4) 3: 353-372.

Cupani, A. (2014). A realidade complexa da tecnologia. Caderno da Unisinos (Brasil).

Ellul, J. (1964). The Technological Society (trad. de La technique ou l'enjeu du siècle, 1954). New York: Vintage Books.

Feenberg, A. (2002). Transforming Technology. A Critical Theory Revisited. (1991). Oxford/New York: Oxford University Press.

Ferré, F. (1995). Philosophy of Technology. (1988). Athens/London: The University of Georgia Press.

Harari, Y. N. (2016a). Sapiens. Uma breve história da humanidade, 18 a ed. (trad. de: Sapiens. A Brief History of Mankind, 2012). Porto Alegre: Ed. L\&PM.

Harari, Y. N. (2016b). Homo Deus. Uma breve história do amanhã. (trad. de: Homo Deus: A Brief History of Tomorrow. Porto Alegre: Ed. L\& PM.

Heidegger, M. (1997). A questão da técnica. São Paulo: Cadernos de tradução da USP, n.2 (trad. de Die Frage nach der Technik, 1954).

Ihde, D. (1990). Technology and the lifeworld: from garden to earth. Bloomington: Indiana University Press.

Lacey, H. (2006). A controvérsia sobre os transgênicos: questôes cientificas e éticas. São Paulo: Ed. Ideias \& Letras. 
Marcuse, H. (1982). A ideologia da sociedade industrial (trad. de One-dimensional man, 1964). Rio de Janeiro: Ed. Zahar.

Mitcham, C. (1994). Thinking through technology: the path between engineering and philosophy. Chicago: The University of Chicago Press.

Mumford, L. (1963). Technics and civilization. (1934). New York: Harcourt Brace.

Ortega y Gasset, J. (1965). Meditación de la Técnica. (1939). Madrid: EspasaCalpe.

Pitt, J.C. (2000). Thinking about technology: foundations of the philosophy of technology. New York: Seven Bridges.

Postman, N. (1993). Technopoly. The surrender of culture to technology. New York: Vintage.

Skolimowski, H. (1983). "The Structure of Thinking in Technology", (orig. 1966), in C. Mitcham and R. Mackey, Philosophy and technology: readings in the philosophical problems of technology. New York: The Free Press: 42-49.

Snow, C.P. (1995). As duas culturas e uma segunda leitura (trad. de The Two Cultures and a Second Look). São Paulo: Ed. da Universidade de São Paulo.

Vincenti W. (1990). What engineers know and how they know it. London: The John Hopkins University.

Winner, L. (1986). "Do Artifacts have Politics?", in L. Winner, The Whale and the Reactor. A Search for Limits in an Age of High Technology. Chicago/ London: The University of Chicago Press: pp. 19-39.

Wittgenstein, L. (1979). Investigaçōes Filosóficas (trad. de Philosophische Untersuchungen, 1953). São Paulo: Ed. Abril. 